\title{
Underutilization of Evidence-based Strategies in the Diagnosis and Treatment of Venous Thromboembolism Among Trainees
}

Vibhu Sharma, mo Charles Koczka, mo Conrad Fischer, MD
Department of Internal Medicine, State University of New York, Downstate Medical Center, Brooklyn, New York.

Disclosure: Nothing to report.

BACKGROUND: The knowledge and attitude of trainees toward the use of prediction rules in the diagnosis of venous thromboembolism (VTE) is understudied. The extent of knowledge as far as imaging strategies in the setting of VTE and use of low molecular weight heparin (LMWH) among trainees is also understudied.

METHODS: This was a cross-sectional study; between October, 2006 and March, 2008, surveys were distributed at grand rounds and national medicine board review courses. Respondents returning completed surveys included 43 medicine attendings, 139 residents, and 134 medical students Emergency physicians were called at work and 46 completed an abbreviated version of the survey. Attending and trainee responses were compared.

RESULTS: Over $60 \%$ of students and $40 \%$ of residents did not use any prediction rules. Most attendings ( $>60 \%$ ) did not use a prediction rule. Among attendings, $48 \%$ of emergency physicians and $30 \%$ of medicine attendings felt that prediction rules were too complex to use. Knowledge about imaging techniques and diagnostic protocols for VTE were worse for students than for residents. A substantial minority of all respondents ( $17 \%$ of students, $12 \%$ of residents and $13 \%$ of medicine attendings) would not use LMWH in the therapy of non-massive pulmonary embolism. In general, level of training did not translate into a greater proportion of correct responses to clinical scenarios or greater knowledge about imaging systems and strategies.

CONCLUSION: Trainees do not use a structured approach to VTE diagnosis. LMWH therapy is underutilized by a large minority of trainees and attendings. A top-down approach is needed to revitalize evidence-based management of VTE. Journal of Hospital Medicine 2010;5:E26-E30. @ 2010 Society of Hospital Medicine.

KEYWORDS: deep vein thrombosis, evidence-based medicine, medical students, pulmonary embolism, residents.

Pretest probability assessment is an important first step in the diagnosis of venous thromboembolism (VTE) and models incorporating Wells criteria ${ }^{1}$ can be used accurately in emergency department (ED) and inpatient settings. ${ }^{2}$ Gestalt has the disadvantage of poor interobserver reliability, ${ }^{3}$ and use of clinical prediction rules has been advocated instead. ${ }^{4}$ In academic institutions, trainees frequently first evaluate patients with suspected VTE, and although gestalt improves with degree of experience, the performance of gestalt in 1 study ${ }^{5}$ was better for attendings than interns or residents (for whom it was equivalent), suggesting that structured pretest probability assessment may be more important for trainees.

From an imaging perspective, multidetector computed tomography (CT), ${ }^{6}$ is more accurate than ventilation perfusion (VP) scanning ${ }^{7,8}$ in diagnosing VTE in any setting, including the critically ill. ${ }^{9,10}$ Lower extremity CT venography (LECTV) has comparable sensitivity to contrast venography and sonography, ${ }^{11}$ and in combination with computed tomographic pulmonary angiography (CTPA) is important when imaging results are discordant with pretest probability. ${ }^{12}$ Guidelines for diagnostic pathways in VTE based on published literature incorporating D-dimer testing have been updated recently, ${ }^{13}$ the degree of adoption and use of diagnostic algorithms among trainees has been understudied.

Clinical trials ${ }^{14,15}$ have confirmed the safety and efficacy of low molecular weight heparin (LMWH) in the treatment of pulmonary embolism (PE) in inpatients, but the degree of adoption of this therapy is unclear. The primary objective of our survey of was to assess the knowledge, attitudes, practices, and preferences of trainees and attendings who order and evaluate the results of diagnostic studies in the management of VTE. A secondary objective was to assess willingness to use LMWH to treat VTE in the inpatient setting among non-ED respondents.

\section{Methods}

\section{Survey Design and Administration}

The study was cross-sectional and was approved by the institutional review board. The survey was paper-based and anonymous, and the requirement for written informed consent was waived. The survey instrument was reviewed for clarity, lack of bias, and accuracy by a panel of hospitalists at the State University of New York (SUNY) Downstate Medical Center. Closed-ended questions were used, including a 5point Likert scale $(1=$ strongly agree, $5=$ strongly disagree $)$ and multiple-choice queries. Between October 2006 and 


\begin{tabular}{lll} 
TABLE 1. Respondent Characteristics & \\
& $\mathbf{n}(\%)$ & $\begin{array}{l}\text { Institutions Rotated } \\
\text { Through [median (IQR)] }\end{array}$ \\
\hline Emergency department attendings & 46 & - \\
Medicine attendings & 46 & $1(1-2)$ \\
Residents & 139 & $3(2-3)$ \\
PGY1 & $39(28)$ & \\
PGY2 & $27(19)$ & \\
PGY3 & $34(24)$ & \\
PGY4 & $3(2)$ & \\
PGY5 & $19(14)$ \\
Year not checked & $17(13)$ \\
Medical students & 134 \\
Third year & $58(43)$ & \\
Fourth year & $76(57)$ \\
\hline Abbreviations: IQR, interquartile range; PGYx, postgraduate year $(\mathrm{x}=1-5)$.
\end{tabular}

\begin{tabular}{|c|c|c|c|}
\hline & $\begin{array}{l}\text { ED Attendings } \\
(\mathrm{n}=46)\end{array}$ & $\begin{array}{l}\text { IM Attendings } \\
(\mathrm{n}=43)\end{array}$ & $P$ Value \\
\hline $\begin{array}{l}\text { Years of experience [median } \\
\text { (interquartile range)] }\end{array}$ & $12.5(7.5-21)$ & $6(2-14)$ & $<0.001$ \\
\hline Academic practice [n (\%)] & $23(50)$ & $27(63)$ & 0.7 \\
\hline $\begin{array}{l}\text { Do not use prediction } \\
\text { rules [n (\%)] }\end{array}$ & $28(61)$ & $28(65)$ & 0.8 \\
\hline $\begin{array}{l}\text { Prediction rules too } \\
\text { complex to use } \\
\text { [n (\% agree)] }\end{array}$ & $22(48)$ & $13(30)$ & 0.5 \\
\hline $\begin{array}{l}\text { Aware of a written } \\
\text { algorithm for diagnosis } \\
\text { of VTE }[n(\%)]\end{array}$ & 2 (4) & $21(50)$ & $<0.001$ \\
\hline
\end{tabular}

March 2008, paper-based survey questionnaires were distributed to internal medicine (IM) attendings, residents, and students from institutions in the New York, New Jersey, and Connecticut tri-state area taking medicine review courses in New York City and attending grand rounds at SUNY Downstate. Of 319 non-ED respondents, 116 (30\%) were from the SUNY Downstate system. All third-year medical students (58/116) were from the SUNY Downstate training program. representing 5 different training institutions for medical students and 4 institutions for residents. ED physicians $(n=46)$ were selected randomly and telephoned at work and questioned about their practices with an abbreviated version of the survey. Response rates were $80 \%$ for the paper-based surveys and $20 \%$ for the ED physicians. Data was recorded into an electronic database (Microsoft Access; Microsoft Corp., Redmond, WA). Simple clinical vignettes were used to assess diagnostic and therapeutic strategies in the setting of VTE for non-ED respondents only.

\begin{tabular}{llllll} 
TABLE 3. Comparison of Responses Assessing & \\
Knowledge Regarding Imaging Studies in VTE & \\
& $\begin{array}{l}\text { ED Attending } \\
(\mathbf{n}=46)\end{array}$ & $\begin{array}{l}\text { IM Attending } \\
(\mathbf{n}=\mathbf{4 3})\end{array}$ & $\begin{array}{l}\text { Residents } \\
(\mathbf{n}=\mathbf{1 3 9})\end{array}$ & $\begin{array}{l}\text { Medical } \\
\text { Students } \\
(\mathbf{n}=\mathbf{1 3 4})\end{array}$ & $\mathbf{P}$ value \\
\hline $\begin{array}{c}\text { VP scanning test } \\
\text { of choice in } \\
\text { suspected }\end{array}$ & $0(0)$ & $9(22)$ & $24(17)$ & $78(58)$ & $<0.001$ \\
$\begin{array}{c}\text { PE [n (\%)] } \\
\text { CTV ordered } \\
\text { separate from } \\
\text { or with CTPA } \\
\text { by default [n } \\
\text { (\% unaware)] }\end{array}$ & & & & \\
$\begin{array}{c}\text { Sensitivity of } \\
\text { CTV = LE US } \\
\text { [n (\% agree)] }\end{array}$ & $12(27)$ & $53(38)$ & $96(72)$ & $<0.001$ \\
\hline
\end{tabular}

Abbreviations: CTPA, computed tomographic pulmonary angiography; CTV, computed tomographic venography; ED, emergency department; IM, internal medicine; LE US, lower extremity ultrasound; MDCT, multi-detector computed tomography; PE, pulmonary embolism; VP, ventilation perfusion; VTE, venous thromboembolism

\section{Data Analysis}

Descriptive statistics were used to report respondents' demographic information and work environments. Data are expressed as proportions, means $\pm \mathrm{SD}$, or medians with interquartile range. Differences in response levels between groups were compared by Fisher's exact test, chi square test, or the Kruskal-Wallis test, where appropriate. Two-sided $P$ values of less than 0.05 were considered significant. Since no difference in the ability of residents and interns to predict PE has been noted, ${ }^{5}$ both groups were analyzed together, as were third-year and fourth-year medical students. JMP version 7.0 software (SAS Institute, Cary, NC) was used to perform all analyses.

\section{Results}

Table 1 lists the characteristics of respondents. Medical attendings reported practicing in up to 5 different institutions, and residents reported rotating through up to 10 different institutions during their residency. Students reported rotating through up to 11 different institutions.

\section{Pretest Probability Assessment}

Table 2 depicts differences between ED and IM attending responses. More than $60 \%$ of all attendings used no structured pretest probability assessment; the rest reported using the Wells criteria. An equivalent proportion of ED and IM attendings thought prediction rules were too complex to use $(P=0.2)$. Years of attending experience did not predict responses regarding perceptions of the complexity of prediction rules $(P=0.5)$. More IM attendings than residents or students felt that prediction rules were too complex for routine use $(P=0.02)$. Among trainees, significantly more 


\begin{tabular}{|c|c|c|c|c|}
\hline & $\begin{array}{l}\text { IM Attending } \\
(\mathrm{n}=46)\end{array}$ & $\begin{array}{l}\text { Residents } \\
(\mathrm{n}=139)\end{array}$ & $\begin{array}{l}\text { Medical } \\
\text { Students } \\
(\mathrm{n}=134)\end{array}$ & $P$ Value \\
\hline $\begin{array}{l}\text { Anticoagulate high } \\
\text { risk patient with } \\
\text { negative CTPA } \\
\text { alone [n (\% agree)] }\end{array}$ & $35(76)$ & $104(75)$ & $99(74)$ & 0.9 \\
\hline $\begin{array}{l}\text { Order further imaging } \\
\text { in outpatient with } \\
\text { negative D-dimer } \\
\text { and low probability } \\
\text { for DVT [n (\% agree)] }\end{array}$ & $25(54)$ & $68(49)$ & $59(44)$ & 0.6 \\
\hline $\begin{array}{l}\text { LMWH appropriate for } \\
\text { DVT and non-massive } \\
\text { PE among inpatients } \\
\text { [n (\% agree)] }\end{array}$ & $40(88)$ & $119(86)$ & $88(66)$ & 0.02 \\
\hline
\end{tabular}

Abbreviations: CTPA, computed tomographic pulmonary angiography; DVT, deep vein thrombosis; IM, internal medicine; LMWH, low molecular weight heparin; PE, pulmonary embolism.

residents than students reported using the Wells model $(P<$ 0.001 ); $40 \%$ of residents did not use any model. Advanced years in training among residents did not predict an increased likelihood of using prediction rules.

\section{D-Dimer Testing}

Among trainees, $25 \%$ of residents and students and $20 \%$ of IM attendings were unaware of the sensitivity or specificity of $\mathrm{D}$-dimer assays in use in their institution $(P=0.8)$, and $70 \%$ of ED attendings were unaware. Almost all residents, students, and IM attendings were unable to identify the name of the D-dimer test used in their institutions $(>95 \%$ in each category); while $54 \%$ of $\mathrm{ED}$ attendings were also unable to do so $(P<0.0001)$.

\section{Imaging Strategies}

Table 3 depicts responses regarding knowledge about various VTE imaging strategies. The majority of students responded that they would use VP scanning as the initial modality and a substantial number of attendings and residents would too. All ED attendings reported using CTPA as the initial modality of choice. A substantial number of students, residents, and IM attendings did not know whether LECTV had to be ordered separately or was done by default and a large proportion incorrectly surmised that the sensitivity of LECTV was not equivalent to lower extremity Doppler.

\section{Clinical Vignettes}

Table 4 depicts responses by non-ED respondents to various clinical scenarios presented. Faced with a dyspneic patient 2 days after a hip fracture and a negative CTPA alone, almost $25 \%$ of all respondents would incorrectly withhold anticoagulation. In outpatients with low probability Wells score for DVT and a negative D-dimer, substantial propor- tions of all respondents would incorrectly order further imaging. For treatment of inpatients with DVT and nonmassive PE, $17 \%$ of students disagreed that $\mathrm{LMWH}$ was appropriate, and similar proportions of residents $(12 \%)$ and IM attendings (13\%) disagreed.

\section{Discussion}

\section{Pretest Probability Assessment}

Our findings that only a minority of trainees and practicing physicians calculate pretest probability using a prediction score translate into potentially inferior (and more costly) care for patients with suspected VTE. This is especially true for academic institutions, where trainees are ordinarily "first responders." Among practitioners in the United States, ${ }^{16}$ $72.5 \%$ prefer an unstructured approach to pretest assessment, whereas $22.9 \%$ use published prediction rules. In this survey, more residents than students or attendings used the Wells criteria for pretest probability testing. The majority of ED attendings surveyed (61\%) used no structured pretest probability assessment, consistent with a retrospective study published recently ${ }^{17}$; however, this may have been because of the relatively experienced group sampled (median number of years in practice was 12.5 compared to 6 years among IM attendings). Students may not be receiving training to use prediction rules because attendings may feel they are too complex to use and/or may not use these rules themselves. A substantial proportion of residents $(40 \%$ in our study) do not use them. Awareness of written algorithms was reported by a minority of all respondents, but did not translate into greater use of prediction rules.

\section{D-Dimer Testing}

Only a few highly sensitive quantitative assays (VIDAS, Tinaquant, Liatest, and Simplired $)^{18-21}$ have been validated in large clinical trials incorporating structured pretest probability assessment and CTPA. Guidelines for diagnosis of VTE recommend that "physicians be informed about the type of $\mathrm{D}$-dimer being used in their practice setting given the substantial variation in D-dimer sensitivity." 22 The sensitivities of quantitative enzyme-linked immunosorbent assays (ELISAs) are clinically and statistically superior to other types of D-dimer tests among patients with VTE. ${ }^{23}$ Over $20 \%$ of all non-ED respondents did not know the sensitivity and specificity of the D-dimer assay in use in their respective institutions and most ( $>70 \%$ in each category) could not name the assay, resulting in potentially inappropriate decision making if nonquantitative ELISA D-dimers were used alone or gestalt were used, especially by trainees.

\section{Imaging Strategies}

Weiss et al. $^{24}$ surveyed U.S. clinicians and found a clear preference for CTPA as the initial imaging modality in patients with suspected PE but did not include the trainee perspective. As level of training progressed, we found a decrease in the percentage of respondents that preferred VP 
scanning over CTPA as the first test of choice; however, $25 \%$ of residents and $17 \%$ of attendings still designated VP scanning as their first choice. The perception of the majority of students in our survey is that VP scanning is the preferred initial test. We conjecture that students do not receive the pertinent training from supervising clinicians in this regard. All ED attendings surveyed used CTPA as their first choice of imaging. Knowledge about whether LECTV was ordered separately from CTPA or done by default was lacking in over $25 \%$ of all non-ED respondents. The lower the level of experience, the more incorrect answers were given. Apropos of the PIOPED II study, ${ }^{12}$ lack of awareness about lower extremity imaging in association with CTPA may therefore contribute to inappropriate decision making, especially in patients with high pretest probability of PE and a negative CTPA alone.

\section{Clinical Vignettes}

Two studies ${ }^{25,26}$ analyzed outcomes in patients with low to intermediate pretest probability PE and negative CTPA alone who did not receive anticoagulation. Both suggest that withholding anticoagulation in these patients is safe. The $25 \%$ of non-ED respondents who would consider withholding anticoagulation in high-risk settings translates into a large number of potentially inappropriate decisions, especially if gestalt is used in pretest probability assessment. This is in line with recommendations from the PIOPED II study that lower extremity imaging and, if necessary, serial lower extremity ultrasonography be performed in high-risk groups. ${ }^{11,12} \mathrm{~A}$ negative validated D-dimer study and a low pretest probability exclude the need for further testing in outpatients with suspected $\mathrm{DVT}^{27}$; however, $50 \%$ of all respondents would order further testing. Thus, regardless of experience, a disparity exists between practice and published literature among both trainees and attendings, especially since further imaging in this setting is not cost effective. ${ }^{28}$

\section{Use of LIMWH}

In a cohort of 946 inpatients in one study, ${ }^{29}$ only $56.1 \%$ of inpatients with DVT or PE were treated with LMWH. In our survey a substantial minority of IM attendings, residents, and students $(12 \%, 13 \%$, and $17 \%$, respectively) would not consider LMWH one of the prefered therapies for VTE in the right clinical setting.

\section{Limitations}

The cross-sectional nature of the survey and localization of non-ED respondents to the New York, New Jersey, and Connecticut tri-state region, limits generalizability to other geographic regions of the country. Responses of ED attendings were sampled nationally. The attendings (ED and IM) sampled were a relatively experienced group (6-12 years of practice) and this may explain the relatively low adoption of prediction rules reflecting the use of gestalt in this group. Additionally, over time, knowledge (and use) of validated D- dimer assays may have increased in the practices evaluated. Among non-ED respondents, 30\% (116/319) were from a single training program (SUNY Downstate) and the responses of these respondents may reflect practice in the institutions sampled, limiting nationwide generalization with the potential for selection bias. The low rate of response from ED physicians $(20 \%)$ was presumably a result of being called at work. We believe the responses are still a valuable insight into the "real-time" practices of the clinicians surveyed and do not preclude a meaningful comparison to the rest of the respondents especially given the significant differences between ED and IM attending knowledge and awareness (Tables 3 and 4).

\section{Conclusions}

Our survey identifies the use of evidence-based strategies in the management of VTE among trainees, a perspective that has been lacking in other studies of physicians in practice. ${ }^{16,24,27}$ Substantial variability in attending practice identified in this survey may impede the adoption of a structured approach to the diagnosis of VTE among trainees, and this survey raises major concerns about mechanisms of diagnosis of VTE. Caprini et al. ${ }^{29}$ believe that "physician knowledge, attitudes, and beliefs are partially responsible for the gap between actual practice and international guidelines." 27 The results of our survey extend this suggestion to trainees and imply that supervisor attitudes may negatively influence trainee practices. Development of written protocols or standardized pathway order sets based on published evidence-based guidelines $^{13}$ in the management of VTE could improve the use of structured pretest probability determination and use of evidence-based strategies among trainees. Finally, comparisons of outcomes using algorithms and usual practice could provide valuable, clinically important data that could inform clinical practice.

\section{Address for correspondence and reprint requests:}

Vibhu Sharma, MD, Box 50, 450 Clarkson Ave, Brooklyn, NY 11203; Telephone: 7182707302; Fax: 7182707304; E-mail: vibhusharmamd@ gmail.com Received 12 February 2009; revision received 29 August 2009; accepted 25 September 2009.

\section{References}

1. Penaloza A, Mélot C, Dochy E, et al. Assessment of pretest probability of pulmonary embolism in the emergency department by physicians in training using the Wells model. Thromb Res. 2007;120(2):173-179.

2. Yap KS, Kalff V, Turlakow A, Kelly MJ. A prospective reassessment of the utility of the Wells score in identifying pulmonary embolism. Med J Aust. 2007;187(6):333-336.

3. Rodger MA, Maser E, Stiell I, Howley HE, Wells PS. The interobserver reliability of pretest probability assessment in patients with suspected pulmonary embolism. Thromb Res. 2005;116(2):101-107.

4. Chunilal SD, Eikelboom JW, Attia J, et al. Does this patient have pulmonary embolism? JAMA. 2003;290(21):2849-2858.

5. Rosen MP, Sands DZ, Morris J, Drake W, Davis RB. Does a physician's ability to accurately assess the likelihood of pulmonary embolism increase with training? Acad Med. 2000;75(12):1199-1205.

6. Wells PS, Anderson DR, Rodger M, et al. Excluding pulmonary embolism at the bedside without diagnostic imaging: management of patients with

2010 Society of Hospital Medicine DOI 10.1002/jhm.631 Published online in wiley InterScience (www.interscience.wiley.com). 
suspected pulmonary embolism presenting to the emergency department by using a simple clinical model and D-dimer. Ann Intern Med. 2001; 135(2):98-107.

7. Blachere H, Latrabe V, Montaudon M, et al. Pulmonary embolism revealed on helical CT angiography: comparison with ventilation perfusion radionuclide lung scanning. AJR Am J Roentgenol. 2000;174:1041-1047.

8. Quiroz R, Kucher N, Zou KH, et al. Clinical validity of a negative computed tomography scan in patients with suspected pulmonary embolism: a systematic review. JAMA. 2005;293(16):2012-2017.

9. Russo V, Piva T, Lovato L, Fattori R, Gavelli G. Multidetector CT: a new gold standard in the diagnosis of pulmonary embolism? State of the art and diagnostic algorithms. Radiol Med. 2005;109(1-2):49-61.

10. Kelly AM, Patel S, Carlos RC, Cronin P, Kazerooni EA. Multidetector row CT pulmonary angiography and indirect venography for the diagnosis of venous thromboembolic disease in intensive care unit patients. Acad Radiol. 2006;13(4):486-495.

11. Cronin P, Weg JG, Kazerooni EA. The role of multidetector computed tomography angiography for the diagnosis of pulmonary embolism. Semin Nucl Med. 2008;38:418-431.

12. Stein PD, Fowler SE, Goodman LR, et al. Multidetector computed tomography for acute pulmonary embolism. N Engl J Med. 2006;354:2317-2327.

13. Institute for Clinical Systems Improvement. Venous Thromboembolism Diagnosis and Treatment. Available at: http://www.icsi.org. Accessed October 2009.

14. The Columbus Investigators. Low-molecular-weight heparin in the treatment of patients with venous thromboembolism. $N$ Engl J Med. 1997;337: $657-662$.

15. Simonneau G, Sors H, Charbonnier B, et al. A comparison of low-molecular-weight heparin with unfractionated heparin for acute pulmonary embolism. The THESEE Study Group. N Engl J Med. 1997;337(10): 663-669.

16. Weiss CR, Haponik EF, Diette GB, Merriman B, Scatarige JC, Fishman EK. Pretest risk assessment in suspected acute pulmonary embolism. Acad Radiol. 2008;15(1):3-14.

17. Smith C, Mensah A, Mal S, Worster A. Is pretest probability assessment on emergency department patients with suspected venous thromboembolism documented before SimpliRED D-dimer testing? CJEM. 2008;10(6): 519-523.

18. van der Graaf F, van den Borne H, van der Kolk M, de Wild PJ, Janssen GW, van Uum SH. Exclusion of DVT with D-dimer testing: comparison of
13 D-dimer methods in 99 outpatients suspected of DVT using venography as a standard. Thromb Haemost. 2000;83:191-198.

19. Perrier A, Roy PM, Sanchez O, et al. Multidetector-row computed tomography in suspected pulmonary embolism. N Engl J Med. 2005;352(17): 1760-1768.

20. Goekoop RJ, Steeghs N, Niessen RW, et al. Simple and safe exclusion of pulmonary embolism in outpatients using quantitative D-dimer and Wells' simplified decision rule. Thromb Haemost. 2007;97(1):146-150.

21. De Monyé W, Sanson BJ, Büller HR, Pattynama PM, Huisman MV; ANTELOPE Study Group. The performance of two rapid quantitative D-dimer assays in 287 patients with clinically suspected pulmonary embolism. Thromb Res. 2002;107(6):283-286.

22. Qaseem A, Snow V, Barry P, et al. Current diagnosis of venous thromboembolism in primary care: a clinical practice guideline from the American Academy of Family Physicians and the American College of Physicians. Ann Fam Med. 2007;5:57-62.

23. Stein PD, Hull RD, Patel KC, et al. D-dimer for the exclusion of acute venous thrombosis and pulmonary embolism. A systematic review. Ann Intern Med. 2004;140:589-602.

24. Weiss CR, Scatarige JC, Diette GB, et al. CT pulmonary angiography is the first-line imaging test for acute pulmonary embolism: a survey of US clinicians. Acad Radiol. 2006;13:434-446.

25. Swensen SJ, Sheedy PF, Ryu JH, et al. Outcomes after withholding anticoagulation from patients with suspected pulmonary embolism and negative computed tomographic findings: a cohort study. Mayo Clin Proc. 2002;77:130-138.

26. Moores LK, Jackson WL Jr., Shorr AF, Jackson JL. Meta-analysis: outcomes in patients with suspected pulmonary embolism managed with computed tomographic pulmonary angiography. Ann Intern Med. 2004;141: 866-874.

27. Wells PS, Owen C, Doucette S, et al. Does this patient have deep vein thrombosis? JAMA. 2006;295(2):199-207.

28. Goodacre S, Sampson F, Stevenson M, et al. Measurement of the clinical and cost-effectiveness of non-invasive diagnostic testing strategies for deep vein thrombosis. Health Technol Assess. 2006; 10(15):1-168.

29. Caprini JA, Tapson VF, Hyers TM, et al. NABOR Steering Committee. Treatment of venous thromboembolism: adherence to guidelines and impact of physician knowledge, attitudes, and beliefs. J Vasc Surg. 2005; 42(4):726-733. 